JIOM Nepal, Volume 41, Number 2, August 2019, page 11-16

\title{
Hemodialysis Catheter-Related Infection in a Teaching Hospital of Central Nepal
}

\author{
${ }^{1}$ Arun Sedhain, ${ }^{2}$ Abja Sapkota, ${ }^{3}$ Narayan B Mahotra \\ 'Department of Medicine (Nephrology Unit), Chitwan Medical College, Bharatpur, Chitwan, Nepal, ${ }^{2}$ Department of \\ Nursing, Nepal Medical College, Jorpati, Kathmandu, Nepal, ${ }^{3}$ Department of Clinical Physiology, Maharajgunj Medical \\ Campus, Institute of Medicine, Maharajgunj, Kathmandu, Nepal
}

\section{Corresponding author:}

Arun Sedhain, MD, DM

Department of Medicine (Nephrology Unit), Chitwan Medical College, Bharatpur, Chitwan, Nepal Email:arunsedhain@gmail.com

Submitted: Jan 5, 2019

Accepted : Jul 20, 2019

\begin{abstract}
Introduction

Infection of the central venous catheter (CVC) is a major complication seen among patients undergoing hemodialysis. Identifying CVC related infection (CRI) and its risk factors and causative organisms is important for better implementation of preventive strategies.
\end{abstract}

\section{Methods}

A prospective study was conducted at Chitwan Medical College for a duration of 2 years from January 2017 to December 2018 among the patients undergoing hemodialysis via CVC. The data collected were related to patients' demographics, site of catheter insertion, and duration, microbiological data including cultures from catheter sites, blood, and catheters' tips and antibiotic sensitivity. Catheter related infection was divided into catheter related local infection (CRLI) and catheter related blood stream infection (CRBSI). Data was analyzed using IBM SPSS Statistics version 21.0.

Results

A total of 41 cases of CVC related infection (CRI) were documented with an incidence rate of 6.94 episodes per 1000 catheter days at risk. Out of the total CRI, 39.02\% were CRLI and 60.98\% were CRBSI. Fever with chills and rigor were the most common clinical presentation. Risk factors for the development of CRI were duration of catheter in situ, repeated change of CVC and the use of CVC for indications other than hemodialysis (HD). Staphylococci and Klebsiella were the most common organisms isolated in culture.

\section{Conclusion}

The rate of CRI among Nepalese patients undergoing hemodialysis is high. Prolonged duration of CVC usage, recent change of catheter and the use of the HD catheter for the purpose of institution of intravenous medication have been found as the risk factors for CRI.

Keywords: Catheter related infection, central venous catheter, hemodialysis

\section{INTRODUCTION}

V ascular access for hemodialysis is attained by arteriovenous fistula (AVF), arteriovenous (AV) graft or central venous catheter (CVC). Central venous catheter (CVC) is used approximately in $80 \%$ of patients at the time of initiation of hemodialysis (HD). ${ }^{1}$ Although CVCs were originally introduced for short-term dialysis, they may be used as a permanent access especially for the patients with limited access options. Dual lumen non-cuffed catheters are used for temporary access whereas dual lumen tunneled catheters are the preferred form of access for intermediate use.
Infection is an important cause of hospitalization and the second most common cause of death among patients undergoing hemodialysis., ${ }^{2,3}$ For clinical convenience, CVC related infection (CRI) has been divided into $\mathrm{CVC}$ related local infection (CRLI) and CVC related bloodstream infection (CRBSI). Hemodialysis catheter-related blood stream infection (CRBSI) remains a significant cause of morbidity and mortality among patients undergoing hemodialysis. ${ }^{4}$ The mean incidence of uncuffed temporary catheters is around 5/1000 catheter days. ${ }^{5}$ In comparison to the arteriovenous (AV) access, the relative risks of catheter related bacteremia and infection-related 
death among patients undergoing hemodialysis has been estimated to be about 10 times and two to three-fold higher respectively. ${ }^{6,7}$ The cumulative likelihood of catheter-related bacteremia has been documented to be 35 percent within three months. ${ }^{8}$

Dialysis catheter-related bacteremia can develop either due to migration from the skin along the outside of the catheter into the bloodstream or due to direct inoculation from a biofilm containing pathogenic micro-organisms. ${ }^{4}$ Multiple factors contribute to the development of dialysis catheter related bacteremia.9,10 The major organisms responsible for such infections are gram-positive organisms, most important of which are coagulase-negative staphylococcal and Staphylococcus aureus. ${ }^{11,12}$

There is paucity of information on the CRI on Nepalese context. So, this study was done with an aim to determine the clinical profile and the microbial etiology of CRBSI among patients with end stage renal disease (ESRD) undergoing hemodialysis in a tertiary care hospital in central part of Nepal.

\section{METHODS}

This prospective study was conducted in the Nephrology unit, Department of Medicine at Chitwan Medical College for a duration of two years from January 2017 to December 2018. Ethical approval was obtained from the Institutional Review Committee of Chitwan Medical College (CMC-IRC) before the initiation of study and written informed consent from the participants was taken.

All patients who were initiated on hemodialysis (HD) using double lumen hemodialysis catheter for more than 48 hours were eligible for inclusion in the study. Patients with CVC insertion for non-dialysis indications and HD patients who presented with any other source of infection were excluded from the study. Participants were followed up for one month after insertion of catheter for any evidence of CRI. Catheter related bacteremia was suspected when a patient experienced fever, chills, purulent discharge from catheter site, erythema or tenderness over exit site, in the absence of any other focus of infection. Blood samples were collected from the hemodialysis catheter hubs and from the peripheral veins using aseptic precaution, which were sent immediately to the laboratory for culture and sensitivity testing. Hemodialysis catheter was removed immediately when clinically indicated, such as CVC infection, nonfunctional CVC, fulfilment of its indication and the death of the patient.Under aseptic precautions, the tip was cut into approximately $5 \mathrm{~cm}$ segment using sterile scissors and transported to the laboratory immediately for culture and sensitivity test. After sending the blood and tip samples for the cultures the patients were immediately started on empiric intravenous antibiotic therapy. For patients who developed a CRBSI, the patient days at risk (catheter days) were defined as the number of days from catheter insertion to the date paired blood cultures were sent. CVC related local infection (CRLI) was diagnosed if a semi quantitative culture of an intravascular catheter segment ( $>15$ colony-forming units) was found to be positive and the diagnosis of CRBSI was based on the clinical presentation of fever, chills and/or hypotension and any of the following two criteria ${ }^{13}$ :

- Same organism recovered from percutaneous blood culture and from semi-quantitative roll-plate culture (>15 CFU) of the catheter tip.

- Same organism recovered from a percutaneous and a catheter lumen blood culture, with growth detected 2 hours earlier (i.e. 2 hours less incubation period) in the blood collected through catheter than the blood collected percutaneously.

Data were entered and analyzed by using IBM SPSS Statistics software version 21.0 (IBM, Armonk, NY). Continuous response variables were presented as mean \pm SD. Chi square test and Fisher exact test were used for qualitative variables. Logistic regression analysis was done to compare the variables between the patients with and without CRI. $\mathrm{p} \leq 0.05$ was considered as statistically significant.

Table 1. Characteristics of study population and CVC catheter

\begin{tabular}{lc}
\hline \multicolumn{1}{c}{ Parameters } & $\begin{array}{c}\text { Number } \\
\text { (Percentage) }\end{array}$ \\
\hline Age (years) & $12(2 \%)$ \\
$<18$ & $138(23.2 \%)$ \\
$18-40$ & $297(50 \%)$ \\
$41-65$ & $147(24.7 \%)$ \\
$>65$ & \\
Sex & $420(70.7 \%)$ \\
Male & $274(29.3 \%)$ \\
Female & \\
Indication for hemodialysis & $210(35.3 \%)$ \\
AKI & $384(64.6 \%)$ \\
CKD & \\
CVC location & \\
Right internal jugular vein & $463(77.9 \%)$ \\
Femoral vein & $118(19.8 \%)$ \\
Left internal jugular vein & $13(2.2 \%)$ \\
Duration of catheterization & \\
$<30$ days & $257(43.2 \%)$ \\
$>30$ days & $337(56.7 \%)$ \\
Reason for CVC catheter removal & \\
No more indicated & $511(86 \%)$ \\
CVC infection & $41(6.9 \%)$ \\
Death of the patient & $25(4.2 \%)$ \\
Not functioning & $17(2.8 \%)$ \\
\hline & \\
\hline & \\
\hline & \\
\hline &
\end{tabular}


Table 2. Clinical features of HD catheter-related infection

\begin{tabular}{lc}
\hline \multicolumn{1}{c}{ Clinical features } & Number (\%) \\
\hline Fever during dialysis & $41(100 \%)$ \\
Chills & $31(75.6 \%)$ \\
Rigors & $31(75.6 \%)$ \\
Nausea, vomiting & $27(65.8 \%)$ \\
Hypotension & $26(63.4 \%)$ \\
Fever before dialysis & $18(43.9 \%)$ \\
Catheter dysfunction & $17(41.4 \%)$ \\
Malaise & $17(41.4 \%)$ \\
Breathlessness & $13(31.7 \%)$ \\
Altered sensorium & $7(17.0 \%)$ \\
Fever after hemodialysis & $5(12.1 \%)$ \\
Erythema at exit site & $5(12.1 \%)$ \\
Purulent discharge & $3(7.3 \%)$ \\
\hline
\end{tabular}

\section{RESULTS}

A total of 594 central venous catheters (CVC) were inserted for the purpose of hemodialysis during the study period. Out of the total patients included in the study, $70.7 \%$ were males. The mean age of the patients was $53.10 \pm 17.75$ years (Range: $16-85$ years).

The indication for the insertion of CVC for hemodialysis was acute kidney injury (AKI) in $35.36 \%$ and chronic kidney disease (CKD) in $64.64 \%$ of patients. HD catheter was kept in situ for a mean period of $9 \pm 4$ days in patients with $A K I$ and $46 \pm 23$ days in patients with CKD. Most commonly used site for CVC insertion was right internal jugular vein (77.94\%), followed by femoral vein $(19.86 \%)$ and left internal jugular vein $(2.2 \%)$. Subclavian vein was used in none of the patients. Details of the baseline parameters is shown on table 1.

A total of 41 cases of CVC related infection (CRI) were documented with an incidence rate of 6.94 episodes per 1000 patient days at risk. Out of the total CRI, 39.02\% were CRLI and $60.98 \%$ were CRBSI. Most common clinical feature was fever with chills and rigor (100\%) followed by nausea and vomiting (25.8\%), Hypotension (63.4\%) and catheter dysfunction (41.4\%). Erythema at infection site and purulent discharge were seen in $12.1 \%$ and $7.3 \%$ of patients respectively. (Table 2 )

Biochemical parameters were compared between the patients with and without CRI, which is shown on table 3. Among patients with $\mathrm{CRI}$, serum level of albumin was lower andthe levels of ferritin and uric acid were significantly higher.

Various possible risk factors were analyzed between the patients with and without CRI. A total of $64.5 \%$ of patients with CKD and $11.9 \%$ of $A K I$ developed CRI. Table 4 shows the different risk factors for the development of CRI.

Most commonly isolated microorganisms in CRI were coagulase negative staphylococci (26.8\%), Staph aureus $(24.4 \%)$ and Klebsiella pneumoniae (21.9\%). Table 5 shows the details of the isolated microorganisms with classification into CRLI and CRBSI.

\section{DISCUSSION}

Even though central venous catheter-related infection (CRI) remains a leading cause of morbidity and mortality among patients undergoing hemodialysis, ${ }^{14}$ initiation of hemodialysis by uncuffed non-tunneled central venous catheter is a common practice especially in south Asian countries. ${ }^{15}$

The incidence rate of $\mathrm{CRI}$ in our study was 6.94 episodes per 1000 patient days at risk, which is higher than the findings from studies from North America, Europe and Africa (ranging between 1.67 and 5.6/1000 catheter days) ${ }^{16-18}$ but slightly lower than that reported from India (7.4 episodes per 1000 catheter days). ${ }^{19}$ One of the reasons for variation in the findings could be the use of different criteria for

Table 3. Laboratory parameters of patients with and without CVC related infection

\begin{tabular}{lccc}
\hline \multirow{2}{*}{ Biochemical parameters } & \multicolumn{2}{c}{ Catheter related infection } & p-value \\
\cline { 2 - 3 } & Positive $(\mathrm{n}=\mathbf{4 1})$ & Negative $(\mathrm{n}=553)$ & \\
\hline WBC count $\left(000 / \mathrm{mm}^{3}\right)$ & $14.6 \pm 6.3$ & $7.6 \pm 3.2$ & 0.35 \\
Hemoglobin $(\mathrm{gm} / \mathrm{dl})$ & $8.9 \pm 2.3$ & $9.45 \pm 1.9$ & 0.14 \\
Platelets $\left(000 / \mathrm{mm}^{3}\right)$ & $134.5 \pm 10.6$ & $186 \pm 11.3$ & 0.96 \\
Albumin $(\mathrm{gm} / \mathrm{dl})$ & $3.0 \pm 0.15$ & $3.4 \pm 0.67$ & 0.04 \\
Iron $(\mu \mathrm{mmol} / \mathrm{L})$ & $24.47 \pm 11.8$ & $15.2 \pm 9.4$ & 0.09 \\
Ferritin $(\mathrm{ng} / \mathrm{mL})$ & $809 \pm 432$ & $506 \pm 205$ & 0.03 \\
TSAT (\%) & $19.4 \pm 6.4$ & $21.34 \pm 7.6$ & 0.08 \\
Uric Acid $(\mathrm{mg} / \mathrm{dL})$ & $8.9 \pm 3.4$ & $6.9 \pm 2.6$ & 0.045 \\
Calcium $(\mathrm{mg} / \mathrm{dL})$ & $9.56 \pm 2.3$ & $9.45 \pm 1.9$ & 1.12 \\
Phosphorus $(\mathrm{mg} / \mathrm{dL})$ & $6.45 \pm 1.7$ & $5.9 \pm 1.6$ & 1.10 \\
PTH & $280 \pm 121$ & $261 \pm 103$ & 0.93 \\
\hline
\end{tabular}

TSAT: transferrin saturation, PTH: parathyroid hormone 
Table 4. Risk factors for CVC related infection (CRI)

\begin{tabular}{|c|c|c|c|c|c|}
\hline \multirow{2}{*}{ Factors } & \multicolumn{2}{|c|}{ Catheter related infection } & \multirow{2}{*}{$\mathrm{HR}$} & \multirow{2}{*}{$95 \% \mathrm{Cl}$} & \multirow{2}{*}{ p-value } \\
\hline & Positive $(n=41)$ & Negative $(n=553)$ & & & \\
\hline Age $>65$ years & $21.9 \%$ & $19.3 \%$ & 1.34 & 0.94-1.65 & 0.90 \\
\hline Male & $73.1 \%$ & $70.3 \%$ & 1.17 & $0.89-1.54$ & 0.95 \\
\hline Diabetes & $17.0 \%$ & $9.5 \%$ & 1.06 & $1.01-1.23$ & 0.08 \\
\hline Femoral CVC & $9.5 \%$ & $6.4 \%$ & 1.92 & $1.56-2.05$ & 0.12 \\
\hline Duration $>30$ days & $78.0 \%$ & $32.1 \%$ & 0.94 & $0.84-1.51$ & 0.04 \\
\hline Recent surgery & $9.7 \%$ & $6.3 \%$ & 1.19 & $0.78-1.57$ & 0.21 \\
\hline Recent change of CVC & $29.2 \%$ & $12.5 \%$ & 0.87 & $0.63-1.08$ & 0.03 \\
\hline Use of CVC for medication & $34.1 \%$ & $5.6 \%$ & 0.89 & $0.52-1.09$ & 0.03 \\
\hline
\end{tabular}

CRI. In this study, IDSA criteria ${ }^{13}$ was used, whereas majority of other studies had used Center for Disease Control (CDC) criteria. $^{20}$

Most common clinical features encountered in this study were fever during dialysis, chills and rigor; nausea and vomiting and hypotension, which are in accordance with other few studies. ${ }^{21-23}$ Significant laboratory parameters among patients with CRI were lower albumin, higher levels of serum ferritin and higher levels of uric acid. Hypoalbuminemia could be an indirect marker of poor nutritional status of the patients undergoing HD and these patients are prone for the development of any infection in the body including CRI. Lower level of albumin can also be seen in systemic infection and inflammation. However, in this study it was difficult to ascertain the cause and effect relationship of serum albumin with CRI. One of the factors hindering the accurate interpretation of the levels of these biochemical parameters could be the timing of the testing in CKD patients undergoing hemodialysis with infection.

The significant risk factors for the development of catheter related infection in this study were the persistence of CVC catheter for a longer duration (>30 days), recent change of CVC with the use of guidewire and the use of hemodialysis catheter for institution of intravenous medication and blood transfusion. Diabetes and prolonged duration of CVC usage have been described as major risk factors related to infection in hemodialysis patients. ${ }^{24-26}$ One of the reasons for the prolonged duration of CVC usage especially in the patients with CKD was due to difficulty of performing an arteriovenous fistula (AVF) which is a common problem encountered in our set up primarily due to reluctancy of the patients and also due to the unavailability of the competent vascular surgeons. To avoid this complication, preparation of AV fistula well in advance in ESRD patients is suggested. Repeated manipulation and changing of dialysis catheter; and the usage of dialysis CVC for the purposes other than dialysis like institution of intravenous medication and blood transfusion needs to be strictly discouraged to avoid further development of CRI.

Although $\mathrm{CRI}$ rate was higher with femoral catheters, location of CVC was not an independent risk factor for $\mathrm{CRI}$ in our study, which is in accordance to few studies ${ }^{26-28}$ but in contrary to the reports by Lemaire et al. ${ }^{29}$ Coagulase negative staphylococci, Staph. aureus and Klebsiella pneumoniae were the most common organisms responsible for CRI. Staph. aureus as the commonest organism causing $\mathrm{CRI}$ has been found in many reports. ${ }^{26,27}$

The reasons for the removal of CVC catheters were the completion of the indication of the catheter, development of CVC infection, death of the patient and non-functional catheter, which were in accordance to the standard guidelines. ${ }^{30}$

Despite exploring the HD catheter related infections in Nepalese context, our study has some limitations. First of all, this was an observational study done in a single center. There was also no control on the length of time the catheters were in place as the lack of

Table 5. Pathogenic organisms causing CVC related infection

\begin{tabular}{lccc}
\hline \multicolumn{1}{c}{ Microorganism } & Overall $(\mathbf{n}=\mathbf{4 1})$ & CRLI $(\mathbf{n = 1 6 )}$ & CRBSI (n=25) \\
\hline Coagulase negative Staphylococci & $11(26.8 \%)$ & $6(37.5 \%)$ & $5(20 \%)$ \\
Staphylococcus aureus & $10(24.4 \%)$ & $1(6.25 \%)$ & $9(36 \%)$ \\
Klebsiella pneumoniae & $9(21.9 \%)$ & $5(31.2 \%)$ & $4(16 \%)$ \\
Proteus species & $4(9.7 \%)$ & $2(12.5 \%)$ & $2(8 \%)$ \\
Escherichia coli & $3(7.3 \%)$ & $1(6.2 \%)$ & $2(8 \%)$ \\
Pseudomonas & $3(7.3 \%)$ & $1(6.2 \%)$ & $2(8 \%)$ \\
Candida species & $1(2.4 \%)$ & 0 & $1(4 \%)$ \\
\hline
\end{tabular}


alternate vascular access influenced the decision to salvage the catheter. Larger multi centric prospective studies are warranted in the future to study the various aspects pertaining to catheter salvageability.

\section{CONCLUSION}

The rate of CRI in Nepalese CKD patients undergoing hemodialysis is high. Prolonged duration of CVC usage, recent change of catheter and the use of the HD catheter for the purpose of institution of intravenous medication have been found as the risk factors for CRI.

\section{ACKNOWLEDGEMENT}

We acknowledge the nursing staff of Hemodialysis Unit at Chitwan Medical College for their help in data collection. We also would like to thank Dr. Bikash Pandey and Mr. Pramuib Ghimire for the compilation of data. We also would like to express our sincere thanks to the patients undergoing hemodialysis at Chitwan Medical College for their consent to participate in the study.

\section{CONFLICT OF INTEREST}

None declared.

\section{REFERENCES}

1. Lok CE, Foley R. Vascular access morbidity and mortality: trends of the last decade. Clin J Am Soc Nephrol. 2013;8(7):1213-9.

2. United States Renal Data System. 2016 USRDS annual data report: Epidemiology of kidney disease in the United States. Bethesda: National Institutes of Health, National Institute of Diabetes and Digestive and Kidney Diseases; 2016. [Internet accessed 2019 July 31].

3. Jaber BL. Bacterial infections in hemodialysis patients: pathogenesis and prevention. Kidney Int. 2005;67:250819.

4. Allon M. Dialysis Catheter-Related Bacteremia: Treatment and Prophylaxis. Am J Kidney Dis. 2004;44:779-91.

5. Kairaitis LK, Gottlieb T. Outcome and complications of temporary haemodialysis catheters. Nephrol Dial Transplant. 1999;14:1710-4.

6. Taylor G, Gravel D, Johnston L, Embil J, Holton D, Paton $S$, et al. Incidence of bloodstream infection in multicenter inception cohorts of hemodialysis patients. Am J Infect Control. 2004;32(3):155-60.

7. Allon M, Daugirdas J, Depner TA, Greene T, Ornt $D$, Schwab SJ. Effect of change in vascular access on patient mortality in hemodialysis patients. Am J Kidney Dis. 2006;47(3):469-77.

8. Lee T, Barker J, Allon M. Tunneled catheters in hemodialysis patients: reasons and subsequent outcomes. Am J Kidney Dis 2005;46(3):501-8.

9. Shingarev R, Barker-Finkel J, Allon M. Natural history of tunneled dialysis catheters placed for hemodialysis initiation. J Vasc Interv Radiol. 2013;24(9):1289-94.

10. Teehan GS, Bahdouch D, Ruthazer R, Balakrishnan VS,
Snydman DR, Jaber BL. Iron storage indices: novel predictors of bacteremia in hemodialysis patients initiating intravenous iron therapy. Clin Infect Dis. 2004;38(8):1090-4

11. Jacobsson G, Dashti S, Wahlberg $T$, Andersson R. The epidemiology of and risk factors for invasive Staphylococcus aureus infections in western Sweden. Scand J Infect Dis 2007; 39:6.

12. Marr KA, Sexton DJ, Conlon PJ, Corey GR, Schwab SJ, Kirkland KB. Catheter-related bacteremia and outcome of attempted catheter salvage in patients undergoing hemodialysis. Ann Intern Med. 1997;127(4):275-80.

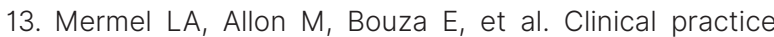
guidelines for the diagnosis and management of intravascular catheter-related infection: 2009 Update by the Infectious Diseases Society of America [published correction appears in Clin Infect Dis. 2010 Apr 1;50(7):1079. Dosage error in article text] [published correction appears in Clin Infect Dis. 2010 Feb 1;50(3):457]. Clin Infect Dis. 2009;49(1):1-45.

14. Jaber BL. Bacterial infections in hemodialysis patients: pathogenesis and prevention. Kidney Int. 2005;67:250819.

15. Hemachandar R. Practice pattern of hemodialysis among end-stage renal disease patients in Rural South India: A single-center experience. Saudi J Kidney Dis Transplant 2017;28:1150-6.

16. Amira CO, Bello BT, Braimoh RW. A study of outcome and complications associated with temporary hemodialysis catheters in a Nigerian dialysis unit. Saudi J Kidney Dis Transplant 2016;27:569-75.

17. Sahli F, Feidjel R, Laalaoui R. Hemodialysis catheterrelated infection: rates, risk factors and pathogens. J Infect Public Health 2017;10: 403-8.

18. Taylor G, Gravel D, Johnston L, Embil J, Holton D, Paton $S$, et al. Incidence of bloodstream infection in multicenter inception cohorts of hemodialysis patients. Am J Infect Control 2004;32:155-60.

19. Agrawal V, Valson AT, Mohapatra A, David VG, Alexander $S$, Jacob $S$, et al. Fast and furious: a retrospective study of catheter-associated bloodstream infections with internal jugular nontunneled hemodialysis catheters at a tropical center. Clinical Kidney Journal. sfy138

20. Centers for Disease Control. Bloodstream Infection Event (Central Line-Associated Bloodstream Infection and Non-central Line Associated Bloodstream Infection). https://www.cdc.gov/nhsn/pdfs/pscmanual/4psc_ clabscurrent.pdf.(Last accessed date: August 3, 2019)

21. Subramanyam TN, Vakrani GP Hemodialysis catheter related blood stream infections. Int J Res Med Sci. 2018 Feb;6(2):562-6.

22. Poole CV, Carlton D, Bimbo L, Allon M. Treatment of catheter-related bacteraemia with an antibiotic lock protocol: effect of bacterial pathogen. Nephrol Dial Transplant. 2004;19(5):1237-44.

23. Miller LM, Clark E, Dipchand C, Hiremath S, Kappel $J$, Kiaii $M$, et al. Hemodialysis tunneled catheterrelated infections. Canadian J kidney Heal Dis. 2016;3:2054358116669129.

24. Oliver MJ, Callery SM, Thorpe KE, Schwab SJ, Churchill DN.Risk of bacteremia from temporary hemodialysis cathetersby site of insertion and duration of use: a prospective study. Kidney Int 2000;58:2543-5.

25. Wang $K$, Wang $P$, Liang $X$, Lu $X$, Liu Z. Epidemiology of haemodialysis catheter complications: a surveyof 865 
dialysis patients from 14 haemodialysis centresin Henan province in China. BMJ Open. 2015;20;5(11):e007136.

26. Sahli F, Feidjel R, Laalaoui R. Hemodialysis catheterrelated infection: rates, risk factors and pathogens. J Infect Public Health. 2017;10(4):403-8.

27. Nabi Z, Anwar S, Barhamein M, Al Mukdad H, El Nassri A. Catheter related infection in hemodialysis patients. Saudi J Kidney Dis Transpl 2009;20(6):1091-5.

28. Parienti JJ, Thirion $M$, Mégarbane $B$, Souweine $B$, OuchikheA, Polito A, et al. Femoral vs jugular venous catheterizationand risk of nosocomial events in adults requiring acute renalreplacement therapy: a randomized controlled trial. JAMA2008;299(20):2413-22.

29. Lemaire $X$, Morena $M$, Leray-Moragues $H$, HenrietViprey D,Chenine L, Defez- Fougeron C, et al. Analysis of risk factorsfor catheter-related bacteremia in 2000 permanent dualcatheters for hemodialysis. Blood Purif 2009;28:21-8.

30. NKF-DOQI Clinical Practice Guidelines for vascular access update. National Kidney Foundation. New York Guideline. 2000;26:25-8. 